\title{
Ortogonalidade de polinômios envolvidos em uma combinação linear com os polinômios de Chebyshev
}

\author{
Mirela Vanina de Mello, \\ Departamento de Ciências Exatas e Tecnológicas, DCET, UESC, \\ 45662-900, Ilhéus, BA \\ E-mail: mirelavanina@gmail.com
}

Resumo: Sejam $\left\{u_{n}\right\}_{n \geq 0}$ e $\left\{S_{n}\right\}_{n \geq 0}$ sequências de polinômios tais que

$$
u_{n}(x)=S_{n}(x)+a_{n-1} S_{n-1}(x), \quad n \geq 1,
$$

onde $\left\{a_{n}\right\}_{n>0} \in \mathbb{R}$ e $u_{n}$ são os polinômios ortogonais de Chebyshev de segunda espécie. Nosso interesse é descobrir quando que $\left\{S_{n}\right\}_{n \geq 0}$ é uma sequência de polinômios ortogonais.

Marcellán e Petronilho [4] resolveram este problema impondo condições sobre os coeficientes $a_{n}$. Eles também obtiveram uma relação entre os funcionais lineares relacionados aos polinômios ortogonais citados. Usando resultados para recuperação da medida de ortogonalidade via determinantes de Turán [5], determinamos tanto a sequência de coeficientes $a_{n}$ para que $\left\{S_{n}\right\}_{n \geq 0}$ seja ortogonal e não apenas o funcional linear, mas sim a função peso com relação a qual os correspondentes polinômios $S_{n}$ são ortogonais. Em outras palavras, a resposta à questão formulada acima foi obtida de uma maneira totalmente diferente e independente da abordagem de Marcellán e Petronilho, sendo a nossa abordagem totalmente analítica enquanto a outra totalmente algébrica.

Palavras-chave: Polinômios Ortogonais, Combinações Lineares de Polinômios, Polinômios de Chebyshev.

\section{Introdução}

Sejam

$$
u_{n}(x)=S_{n}(x)+a_{n-1} S_{n-1}(x), \quad n \geq 1,
$$

onde $a_{n} \in \mathbb{R}, n \geq 0$ e $u_{n}$ são os polinômios ortonormais de Chebyshev de segunda espécie. Sabemos que estes polinômios são ortonormais com relação à função peso

$$
\frac{2}{\pi} \sqrt{1-x^{2}}
$$

no intervalo $(-1,1)$ e que satisfazem a relação de recorrência de três termos

$$
u_{n}(x)=2 x u_{n-1}(x)-u_{n-2}(x), \quad n \geq 2,
$$

com $u_{0}(x)=1$ e $u_{1}(x)=2 x$. Para mais detalhes sobre esses polinômios consulte, por exemplo, o texto clássico de Chihara, [1].

Neste trabalho, encontraremos condições suficientes sobre os coeficientes $a_{n}, n \geq 0$, para que $\left\{S_{n}\right\}_{n \geq 0}$ seja uma sequência de polinômios ortogonais com relação à uma certa medida $d \phi$. Em seguida, apresentaremos a forma explícita de $d \phi$. Em outras palavras, demonstraremos aqui o seguinte teorema: 
Teorema 1.1. Se

$$
\left\{\begin{array}{l}
a_{n}=\frac{a_{n-1}}{a_{n-1}\left(a_{n-2}-a_{n-1}\right)+1}, \quad n \geq 2, \\
\left|a_{0}\right|<1 / 2,
\end{array}\right.
$$

então $\left\{S_{n}\right\}_{n \geq 0}$ é uma sequência de polinômios ortogonais com relação à medida

$$
d \phi(x)=\frac{4 a_{0}}{\pi}\left(x+\frac{1}{2 a_{0}}\right) \sqrt{1-x^{2}} d x,
$$

no intervalo $(-1,1)$.

Nossa abordagem consistirá em utilizar o seguinte resultado, encontrado no trabalho de Máté, Nevai e Totik [5].

Teorema 1.2. Dada uma sequência de polinômios ortonormais $\left\{p_{n}\right\}_{n \geq 0}$ que satisfaz a relação de recorrência de três termos

$$
\delta_{n+1} p_{n}(x)=\left(x-\beta_{n}\right) p_{n-1}(x)-\delta_{n} p_{n-2}(x)
$$

tal que os coeficientes dessa relação satisfazem às condições de Nevai

$$
\lim _{n \rightarrow \infty} \delta_{n}=\frac{1}{2}, \quad \lim _{n \rightarrow \infty} \beta_{n}=0
$$

e são de variação limitada, isto é,

$$
\sum_{n=0}^{\infty}\left|\delta_{n+2}-\delta_{n+3}\right|+\left|\beta_{n+1}-\beta_{n+2}\right|<\infty,
$$

então $d \phi(x)=w(x) d x$, com $w(x)$ estritamente positiva e contínua em $(-1,1)$. Além disso

$$
\lim _{n \rightarrow \infty}\left[\left[p_{n}(x)\right]^{2}-p_{n+1}(x) p_{n-1}(x)\right]=\frac{2}{\pi} \frac{\sqrt{1-x^{2}}}{w(x)}
$$

uniformemente em todo subconjunto compacto de $(-1,1)$.

\section{Ortogonalidade dos polinômios $S_{n}$}

Através da relação (2), substituindo cada polinômio $u_{n}$ pela combinação linear de $S_{n}$ e $S_{n-1}$ dada por (1), obtemos o seguinte resultado:

Lema 2.1. Os polinômios $S_{n}$ podem ser obtidos através da relação de recorrência de quatro termos:

$$
S_{n}(x)=\left(2 x-a_{n-1}\right) S_{n-1}(x)+\left(2 x a_{n-2}-1\right) S_{n-2}(x)-a_{n-3} S_{n-3}(x),
$$

para $n \geq 2$, onde $S_{-1}(x)=a_{-1}=0, S_{0}(x)=1$ e $S_{1}(x)=2 x-a_{0}$.

Usando o lema anterior, podemos estabelecer uma outra relação para os polinômios $S_{n}$.

Lema 2.2. Para $n \geq 4$, os polinômios $S_{n}$ satisfazem

$$
\begin{aligned}
S_{n}(x)= & {\left[2 x-a_{n-1}+a_{n-2}\right] S_{n-1}(x)-\gamma_{n} S_{n-2}(x)-\left(a_{n-3}-a_{n-2} \gamma_{n-1}\right) S_{n-3}(x) } \\
& +\sum_{j=1}^{n-3}(-1)^{n-j-1} a_{j+1} a_{j+2} \ldots a_{n-3} a_{n-2}\left(a_{j-1}-a_{j} \gamma_{j+1}\right) S_{j-1}(x),
\end{aligned}
$$


onde $\gamma_{n}=\left[a_{n-2}\left(a_{n-3}-a_{n-2}\right)+1\right]$, para $n \geq 2$ com $a_{-1}=0$, sendo

$$
\begin{aligned}
& S_{3}(x)=\left[2 x-a_{2}+a_{1}\right] S_{2}(x)-\gamma_{3} S_{1}(x)-\left(a_{0}-a_{1} \gamma_{2}\right) S_{0}(x), \\
& S_{2}(x)=\left[2 x-a_{1}+a_{0}\right] S_{1}(x)-\gamma_{2} S_{0}(x), \\
& S_{1}(x)=\left[2 x-a_{0}\right] S_{0}(x)
\end{aligned}
$$

$e S_{0}(x)=1$.

Note que se $a_{j-1}-a_{j} \gamma_{j+1}=0$ para $j \geq 1$, isto é, se

$$
a_{j}=\frac{a_{j-1}}{a_{j-1}\left(a_{j-2}-a_{j-1}\right)+1}, \quad j \geq 2,
$$

com $a_{1}=\frac{a_{0}}{1-a_{0}^{2}}$, pela relação $(6)$, temos

$$
S_{n}(x)=\left[2 x-a_{n-1}+a_{n-2}\right] S_{n-1}(x)-\gamma_{n} S_{n-2}(x),
$$

com $S_{0}(x)=1$ e $S_{1}(x)=\left(2 x-a_{0}\right) S_{0}(x)$. Assim, os polinômios $S_{n}$ satisfazem a uma relação de recorrência de três termos, com $\gamma_{n}>0$. Logo, pelo clássico Teorema de Favard (consulte, por exemplo, [1]), $\left\{S_{n}\right\}_{n \geq 0}$ é uma sequência de polinômios ortogonais.

A seguir enunciaremos alguns resultados sobre os coeficientes $a_{n}$ que serão de extrema importância para obtermos a medida de ortogonalidade de $S_{n}$.

Primeiramente, usando a condição (7) para os coeficientes $a_{n}$ e o princípio da indução finita, obtemos as seguintes representações para tais coeficientes:

Lema 2.3. Seja $\left\{a_{n}\right\}_{n \geq 0}$ uma sequência de coeficientes que satisfazem à relação (7). Então,

$$
a_{2 m}=a_{0} \frac{Q_{m}\left(a_{0}^{2}\right)}{R_{m}\left(a_{0}^{2}\right)} \quad \text { e } \quad a_{2 m+1}=a_{0} \frac{R_{m}\left(a_{0}^{2}\right)}{Q_{m+1}\left(a_{0}^{2}\right)}, \quad m \geq 0,
$$

onde

$$
\begin{aligned}
& Q_{m+1}\left(a_{0}^{2}\right)=-a_{0}^{2} Q_{m}\left(a_{0}^{2}\right)+R_{m}\left(a_{0}^{2}\right), \\
& R_{m+1}\left(a_{0}^{2}\right)=-a_{0}^{2} Q_{m}\left(a_{0}^{2}\right)+\left(1-a_{0}^{2}\right) R_{m}\left(a_{0}^{2}\right),
\end{aligned}
$$

com $Q_{0}=R_{0}=1$. Em particular, se $a_{0}=0$, então $S_{n}(x)=u_{n}(x), n \geq 1$.

Lema 2.4. Seja $\left\{a_{n}\right\}_{n \geq 0}$ uma sequência de coeficientes que satisfazem à relação (7). Então,

$$
\begin{aligned}
a_{2 m} & =a_{0} \frac{\left(1+d_{0}\right)\left(1-2 a_{0}^{2}+d_{0}\right)^{m}-\left(1-d_{0}\right)\left(1-2 a_{0}^{2}-d_{0}\right)^{m}}{\left(1-2 a_{0}^{2}+d_{0}\right)^{m+1}-\left(1-2 a_{0}^{2}-d_{0}\right)^{m+1}}, \\
a_{2 m+1} & =\frac{2 a_{0}\left[\left(1-2 a_{0}^{2}+d_{0}\right)^{m+1}-\left(1-2 a_{0}^{2}-d_{0}\right)^{m+1}\right]}{\left(1+d_{0}\right)\left(1-2 a_{0}^{2}+d_{0}\right)^{m+1}-\left(1-d_{0}\right)\left(1-2 a_{0}^{2}-d_{0}\right)^{m+1}},
\end{aligned}
$$

com $d_{0}=\sqrt{1-4 a_{0}^{2}}$.

Demonstração: Note que as relações (9) e (10) formam um sistema de equações de diferenças. Para encontrarmos a forma explícita para $R_{m}$ e $Q_{m}$, basta encontrar a solução desse sistema e substituirmos a solução no lema anterior.

Para encontrarmos a medida com relação à qual $S_{n}$ é ortogonal, precisamos transformar os polinômios ortogonais $S_{n}$ em polinômios ortonormais, para tal abordagem nos referenciamos à [3]. Vamos denotar por

$$
s_{n}(x)=\left(\prod_{k=0}^{n-1}\left[a_{k}\left(a_{k-1}-a_{k}\right)+1\right]\right)^{-1 / 2} S_{n}(x),
$$


com $s_{0}(x)=1$ e $a_{-1}=0$, o polinômio $S_{n}$ ortonormalizado. Definindo

$$
\pi_{n}:=\left(\prod_{k=0}^{n-1}\left[a_{k}\left(a_{k-1}-a_{k}\right)+1\right]\right)^{1 / 2}
$$

obtemos a nova combinação linear entre os polinômios ortonormais $u_{n}$ e $s_{n}$

$$
\pi_{n} s_{n}(x)+a_{n-1} \pi_{n-1} s_{n-1}(x)=u_{n}(x) .
$$

Além disso, os polinômios ortonormais $s_{n}$ satisfazem a relação de recorrência de três termos

$$
\frac{\sqrt{a_{n-1}\left(a_{n-2}-a_{n-1}\right)+1}}{2} s_{n}(x)=\left(x-\frac{a_{n-1}-a_{n-2}}{2}\right) s_{n-1}(x)-\frac{\sqrt{a_{n-2}\left(a_{n-3}-a_{n-2}\right)+1}}{2} s_{n-2}(x),
$$

com $s_{0}(x)=1$ e $s_{1}(x)=\frac{2 x-a_{0}}{\sqrt{1-a_{0}^{2}}}$.

Dada a forma explícita para os coeficientes $a_{n}$, obtemos as seguintes expressões limites:

Lema 2.5. Para

$$
\left|a_{0}\right|<\frac{1}{2}
$$

com $a_{0} \neq 0$,

$$
\lim _{n \rightarrow \infty} a_{n}=\frac{1-d_{0}}{2 a_{0}} \quad e \quad \lim _{n \rightarrow \infty} \pi_{n}=\frac{\sqrt{2} a_{0}}{\sqrt{1-d_{0}}} .
$$

Utilizando os resultados anteriores e algumas manipulações algébricas, não é difícil mostrar que as hipóteses do Teorema 1.2 são todas satisfeitas. Assim, de posse de todos os resultados apresentados, podemos demonstrar o teorema principal deste trabalho.

Teorema 2.1. Seja

$$
\pi_{n} s_{n}(x)+a_{n-1} \pi_{n-1} s_{n-1}(x)=u_{n}(x), \quad n \geq 1,
$$

onde $\left\{u_{n}\right\}$ denota a sequência de polinômios ortonormais de Chebyshev de segunda espécie. Se

$$
a_{n}=\frac{a_{n-1}}{a_{n-1}\left(a_{n-2}-a_{n-1}\right)+1}, \quad n \geq 1,
$$

com $a_{-1}=0$, então a sequência $\left\{s_{n}\right\}$ também é uma sequência de polinômios ortogonais. Além disso, denotando por d $\phi$ a medida com relação à qual $s_{n}$ é ortonormal, se

$$
\left|a_{0}\right|<\frac{1}{2}
$$

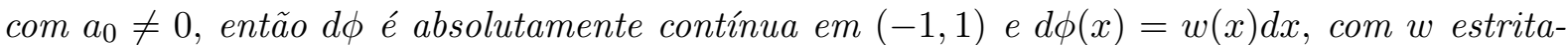
mente positiva e contínua em $(-1,1)$. Ademais, a função peso $w(x)$ é dada por

$$
w(x)=\frac{4 a_{0}}{\pi}\left(x+\frac{1}{2 a_{0}}\right) \sqrt{1-x^{2}} .
$$

Demonstração: Usando (15), obtemos

$$
\begin{aligned}
& u_{n}^{2}(x)-u_{n+1}(x) u_{n-1}(x)=\left[\pi_{n} s_{n}(x)+a_{n-1} \pi_{n-1} s_{n-1}(x)\right]^{2} \\
&-\left[\pi_{n+1} s_{n+1}(x)+a_{n} \pi_{n} s_{n}(x)\right]\left[\pi_{n-1} s_{n-1}(x)+a_{n-2} \pi_{n-2} s_{n-2}(x)\right] .
\end{aligned}
$$


Fazendo $n$ tender ao infinito em (16) e usando o Lema 2.5, obtemos

$$
\begin{aligned}
& \lim _{n \rightarrow \infty}\left[u_{n}^{2}(x)-u_{n+1}(x) u_{n-1}(x)\right]=\frac{2 a_{0}^{2}}{1-d_{0}} \lim _{n \rightarrow \infty}\left[s_{n}^{2}(x)-s_{n+1}(x) s_{n-1}(x)\right] \\
& \quad+\frac{1-d_{0}}{2} \lim _{n \rightarrow \infty}\left[s_{n-1}^{2}(x)-s_{n}(x) s_{n-2}(x)\right]+a_{0} \lim _{n \rightarrow \infty}\left[s_{n}(x) s_{n-1}(x)-s_{n+1}(x) s_{n-2}(x)\right] .
\end{aligned}
$$

Do Teorema 1.2, podemos afirmar que

$$
\lim _{n \rightarrow \infty}\left[s_{n}^{2}(x)-s_{n+1}(x) s_{n-1}(x)\right]=\frac{2}{\pi} \frac{\sqrt{1-x^{2}}}{w(x)} .
$$

Ademais, utilizando um resultado semelhante ao Teorema 1.2, encontrado em [2], e sabendo que $u_{2}(x)=4 x^{2}-1$ e $u_{0}^{(1)}(x)=1$, o polinômio associado, obtemos

$$
\begin{aligned}
\lim _{n \rightarrow \infty}\left[s_{n}(x) s_{n-1}(x)-s_{n+1}(x) s_{n-2}(x)\right] & =\frac{2}{\pi} \frac{\sqrt{1-T^{2}(x)}}{w(x)} \operatorname{sinal}\left[T^{\prime}(x)\right] \\
& =\frac{4|x|}{\pi} \frac{\sqrt{1-x^{2}}}{w(x)} \frac{x}{|x|},
\end{aligned}
$$

onde

$$
T(x)=\frac{1}{2}\left(u_{2}(x)-u_{0}^{(1)}(x)\right)=2 x^{2}-1 .
$$

Assim, utilizando as igualdades (17), (18) e (19) e a identidade para os polinômios ortonormais de Chebyshev

$$
u_{n}^{2}(x)-u_{n+1}(x) u_{n-1}(x)=1,
$$

obtemos

$$
\begin{aligned}
1=\lim _{n \rightarrow \infty}\left[u_{n}^{2}(x)-u_{n+1}(x) u_{n-1}(x)\right]= & \frac{2 a_{0}^{2}}{1-d_{0}} \frac{2}{\pi} \frac{\sqrt{1-x^{2}}}{w(x)}+\frac{1-d_{0}}{2} \frac{2}{\pi} \frac{\sqrt{1-x^{2}}}{w(x)} \\
& +a_{0} \frac{4 x}{\pi} \frac{\sqrt{1-x^{2}}}{w(x)} \\
= & \frac{4}{\pi} \frac{\sqrt{1-x^{2}}}{w(x)} a_{0}\left(x+\frac{1}{2 a_{0}}\right) .
\end{aligned}
$$

Portanto, obtemos o resultado desejado, ou seja,

$$
w(x)=\frac{4 a_{0}}{\pi}\left(x+\frac{1}{2 a_{0}}\right) \sqrt{1-x^{2}} .
$$

Observe que $w(x) \geq 0$, mas não identicamente nula em $(-1,1)$ se, e somente se, $\left|a_{0}\right|<\frac{1}{2}$.

\section{Referências}

[1] T.S. Chihara, "An introduction to orthogonal polynomials", Gordon and Breach, New York, 1978.

[2] J.S. Geronimo; W. Van Assche, Approximating the weight function for orthogonal polynomials on several intervals, J. Approx. Theory, 65 (1991) 341-371.

[3] M.H. Ismail; M.E. Muldoon, A discrete approach to monotonicity of zeros of orthogonal polynomials, Trans. Amer. Math. Soc. 323 (1991) 65-78. 
[4] F. Marcellán; J. Petronilho, Orthogonal polynomials and coherent pairs: the classical case, Indag. Mathem. 6 (1995), 287-307.

[5] A. Máté; P. Nevai; V. Totik, Asymptotics for orthogonal polynomials defined by a recurrence relation, Constr. Approx. 1 (1985) 231-248. 